\title{
TARGET SPACE SUPERSYMMETRIC SIGMA MODEL TECHNIQUES*
}

\author{
JAN DE BOER ${ }^{\dagger}$ \\ Department of Physics, University of California at Berkeley \\ 366 LeConte Hall, Berkeley, CA 94720-7300, USA \\ and \\ Theoretical Physics Group, Mail Stop 50A-5101 \\ Ernest Orlando Lawrence Berkeley National Laboratory, Berkeley, CA 94720, USA \\ KOSTAS SKENDERIS ${ }^{\ddagger}$ \\ Instituut voor Theoretische Fysica, Katholieke Universiteit Leuven \\ Celestijnenlaan 200D, B-3001 Leuven, Belgium
}

\begin{abstract}
We briefly review the covariant formulation of the Green-Schwarz superstring by Berkovits, and describe how a detailed tree-level and one-loop analysis of this model leads, for the first time, to a derivation of the low-energy effective action of the heterotic superstring while keeping target-space supersymmetry manifest. The resulting low-energy theory is old-minimal supergravity coupled to tensor multiplet. The dilaton is part of the compensator multiplet.
\end{abstract}

\section{Introduction.}

Sigma model techniques provide the most effective way of obtaining the low energy effective actions of string theories. The latter are of fundamental importance because they do not only provide the starting point for string-inspired phenomenology but also set up the right framework to address theoretical questions such as the occurence of anomalies or the existense of duality symmetries. Furthermore, target space effective actions may turn out to be a powerful tool to understand the dynamics of $M$ (or $F$ ) theory by yielding worldsheet actions for theories of membranes and other extended objects 1 .

Two dimensional sigma models describe the coupling of string theory to background fields that are the dynamical fields of the corresponding low energy

\footnotetext{
* preprint no:LBNL-39561, UCB-PTH-96/48, KUL-TF-96/22. To appear in proceedings of workshop on Gauge Theory, Applied Supersymmetry and Gauge Theory, Imperial College, July 5-10 1996, and in the e-proceedings of Strings'96, Santa Barbara, July 15-20 1996 , and Argonne Duality Institute, June 27-July 121996.

†e-mail:Deboer@theOr3.LBL.gOv. Fellow of the Miller Institute for Basic Research in Science; supported in part by the Director, Office of Energy Research, Office of Basic Energy Services, of the US Department of Energy under Contract DE-AC03-76SF00098 and in part by the National Science Foundation under grant PHY95-14797.

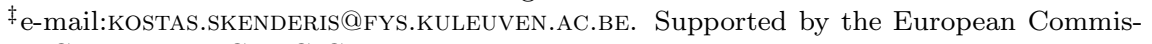
sion HCM program CHBG-CT94-0734.
} 
effective field theory. Standard arguments show that the conformal invariance of the sigma model yields the field equations of the background fields. However, until recently, only bosonic background fields could be coupled to sigma models. The determination of the fermion terms in the effective action were left to (more cumbersome) $S$-matrix techniques. This imposes certain limitations on the sigma model methods since the supersymmetrization of bosonic actions is often neither straightforward nor unique. Ideally, one would like to have a formulation that is manifestly covariant under all symmetries that the target space theory may have. In particular, one would like to keep manifest the target space supersymmetry if present. This would be achieved by having a formulation directly in terms of target superspace. This formulation would then yield the off-shell description of the low energy effective theory. An off-shell description is important for a variety of reasons. It constrains the allowed types of matter coupling in the low energy effective action, it provides constraints on the structure of the higher order $\alpha^{\prime}$-corrections, and it provides the natural framework to study the interplay between dualities and supersymmetry, supersymmetry breaking, and non-renormalization theorems. In addition, it would be preferable to have all SUSY auxiliary fields present if the target space action is going to be a worldsheet action of another theory.

A manifestly target space supersymmetric formulation of superstrings, known as Green-Schwarz formalism, has been knownl for more than a decade. The problem, however, of quantizing this string while keeping all target space symmetries manifest remained unsolved for a long period, thus preventing the development of manifestly supersymmetric sigma model techniques. It was only after the breakthrough due to Berkovits $\mathrm{B}$ that such calculations were made possible at all. Berkovits managed to find a formulation that can be used to covariantly quantize $4 \mathrm{~d}$ compactifications of heterotic and type II strings. His model contains $N=2$ world-sheet supersymmetry, is related by a redefinition to the standard $N=1 R N S$ description and reduces to the standard light cone Green-Schwarz formulation upon gauge fixing. Following up, Berkovits and Siegell constructed the corresponding sigma models. Indirects arguments based om symmetry considerations give the general form of the low energy effective action. The precise form of the latter, however, only follows from an explicit "beta function" calculation. It is important to perform such a calculation since not only it pinpoints the precise low energy model which is of interest for phenomenology but also it provides very strong evidence that the structure encoded in the corresponding sigma model is the correct one. A contraversial issue which is resolved with the new supersymmetric techniques is to which supermultiplet the dilaton belongs. A new paradigm seems to emerget according to which the dilaton is always part of the compensator multiplets. 
This is true in the bosonic string (the kinetic term of the dilaton comes with the wrong sign in the low energy effective action), and it has been verified by us 6 to be also true for the heterotic string. In the latter case, the dilaton is shown to be part of a chiral multiplet and not of a linear multiplet as it was erroneously assumed in the past 0 . The point here is that one should correctly identify the dilaton. We take the dilaton to be by definition the worldsheet field that counts string loops. This means that it couples to the world-sheet curvature in the sigma model. Then one can correctly identify the multiplet to which the dilaton belongs by following the derivation of the low energy effective action. In the case of type II strings there are two compensators, one which is chiral (a vector multiplet) and another which is twisted-chiral (a tensor hypermultiplet). The Euler number couples to the sum of the a vector and a tensor compensator 4 . This contradicts the standard folklore that the dilaton is sitting solely in a hypermultiplet. We intend to settle this issue in a future publication 8 by an explicit calculation of the type described in this contribution.

Recently we performed a detailed study of the heterotic sigma model 6 . We explicitely checked the superconformal invariance up to one-loop in $\alpha^{\prime}$ by perturbatively computing the OPE's of the generators of the $N=2$ superconformal algebra in the supergravity background. The tree-level superconformal invariance yielded the complete supergravity algebra, and the one-loop superconformal invariance the equations of motion of the low energy effective theory. We then integrated these equation to obtain the low energy effective action. The resulting low energy effective theory is old-minimal supergravity coupled to a tensor multiplet.

In this contribution we will give some details of the "conventional" beta function calculation. This calculation is much simpler than the diagramatic evaluation of the OPE's. Checking the finiteness of the sigma model, however, is in general a weaker condition that the condition for superconformal invariance.

\section{The supersymmetric sigma model}

In this section we briefly review the supersymmetric sigma model 6 for the heterotic string compactified to four dimensions. The action reads

$$
\begin{gathered}
S=\frac{1}{\alpha^{\prime}} \int d^{2} z\left(\frac{1}{2} \Pi^{\alpha \dot{\alpha}} \bar{\Pi}_{\alpha \dot{\alpha}}+d_{\alpha} \bar{\Pi}^{\alpha}+d_{\dot{\alpha}} \bar{\Pi}^{\dot{\alpha}}+\frac{1}{2} \bar{\Pi}^{A} \bar{\Pi}^{B} B_{B A}\right. \\
\left.-\frac{\alpha^{\prime}}{2}(\bar{\partial} \rho+i \bar{\partial}(\phi-\bar{\phi}))\left(\partial \rho+i \partial(\phi-\bar{\phi})+a_{z}\right)\right)
\end{gathered}
$$


Here, $\Pi^{A}=\partial z^{M} E_{M}{ }^{A}, \bar{\Pi}^{A}=\bar{\partial} z^{M} E_{M}{ }^{A}$, where $z^{M}=\left(x^{M}, \theta^{\alpha}, \bar{\theta}^{\dot{\alpha}}\right)$ are the coordinates of flat $4 \mathrm{~d}$ superspace, and $E_{M}{ }^{A}$ is the supervielbein. The other target space superfields are the super two-form $B_{B A}$ and the dilaton fields $\phi$ and $\bar{\phi}$ that are chiral and anti-chiral superfields. The fields $d_{\alpha}, d_{\dot{\alpha}}$ are anti-commuting world-sheet fields of conformal weight $(1,0)$, and $\rho$ is a boson subject to the constraint $\bar{\partial} \rho+i \bar{\partial}(\phi-\bar{\phi})=0$, which is imposed by the Lagrange multiplier $a_{z}$. In principle, we still have to add to (11) a piece containing the compactification dependent states and the Yang-Mills fields, but we will restrict our attention to the pure $4 \mathrm{~d}$ superfields only.

One crucial feature of (11) is that it is manifestly target-space supersymmetric. In addition, the dilaton is part of a separate superfield and does not belong to the same superfield as the antisymmetric tensor, in contrast to what is sometimes claimed. The way the dilaton enters is dictated by the world-sheet symmetries of (1). In the anti-holomorphic sector, it has a Virasoro algebra, but in the holomorphic sector it has an $N=2$ algebra. The dilaton enters by means of a generalization of the Fradkin-Tseytlin term 1 to $N=(2,0)$ worldsheet supergravity. The $N=2$ algebra of the sigma model can be used to prove its equivalence with the usual RNS formulation of the heterotic string. The BRST cohomology of the sigma model in flat space yields the same spectrum as that of the conventional RNS string, and to first order in the background fields the sigma model is given by its flat space form plus the corresponding vertex operators. This demonstrates that it correctly describes the heterotic string. The sigma model looks somewhat similar to the Green-Schwarz superstring, however there are important differences. The sigma model given above does not have $\kappa$-symmetry, and it turns out that the background fields are not put on-shell already at tree-level. Without this property, the sigma model would not be a proper off-shell description of the heterotic string. In addition, in (便) one finds the chiral boson $\rho+i(\phi-\bar{\phi})$, which does complicate matters somewhat.

The $N=2$ algebra of (11) has generators

$$
\begin{aligned}
J= & -i \partial \rho+\partial \phi-\partial \bar{\phi} \\
G= & \frac{1}{i \alpha^{\prime} \sqrt{8 \alpha^{\prime}}} e^{i \rho} d^{\alpha} d_{\alpha}-\frac{1}{\sqrt{2 \alpha^{\prime}} i} \partial\left(e^{i \rho} d^{\gamma} \nabla_{\gamma} \phi\right) \\
\bar{G}= & \frac{1}{i \alpha^{\prime} \sqrt{8 \alpha^{\prime}}} e^{-i \rho} d^{\dot{\alpha}} d_{\dot{\alpha}}-\frac{1}{\sqrt{2 \alpha^{\prime} i}} \partial\left(e^{-i \rho} d^{\dot{\gamma}} \nabla_{\dot{\gamma}} \bar{\phi}\right) \\
T= & \frac{1}{\alpha^{\prime}}\left(-\frac{1}{2} \Pi^{\alpha \dot{\alpha}} \Pi_{\alpha \dot{\alpha}}-d_{\alpha} \Pi^{\alpha}-d_{\dot{\alpha}} \Pi^{\dot{\alpha}}\right. \\
& \left.+\frac{\alpha^{\prime}}{2} \partial(\rho+i(\phi-\bar{\phi})) \partial(\rho+i(\phi-\bar{\phi}))\right)-\frac{1}{2} \partial^{2}(\phi+\bar{\phi}) .
\end{aligned}
$$


In the case of the bosonic string, where one only has a Virasoro algebra, the equations of motion of the low-energy effective action follow by requiring that the sigma model is conformal. This can be checked by computing its betafunctions. In our case, we want something stronger, namely that (11) is $N=2$ superconformal. Since it is not possible to write (11) as an action in $N=(2,0)$ world-sheet superspace, a conventional beta-function calculation would only guarantee conformal invariance. In order to check to full $N=2$ superconformal invariance, we used a different method, first applied to the bosonic string by Banks et al.10. This method consists of a direct computation of the operator product expansions of the generators of the $N=2$ algebra, using a covariant background field formalism. In the case of the bosonic string, no information is obtained classically and at one loop one obtains the equations of motion of the low-energy effective field theory. In our case, we do obtain a set of constraints classically, that reduce the field content from the supervielbein $E$ and the super two-form $B$ to that of conformal supergravity coupled to a tensor multiplet. These constraints are similar to the so-called beta-function favored constraints 11 . Together with the Bianchi identities they completely determine the supergravity algebra, with the result

$$
\begin{aligned}
\left\{\nabla_{\alpha}, \nabla_{\beta}\right\}= & 0, \\
\left\{\nabla_{\alpha}, \nabla_{\dot{\beta}}\right\}= & -2 i \nabla_{\alpha \dot{\beta}}-4 i H_{\dot{\beta} \gamma} M_{\alpha}^{\gamma}+4 i H_{\dot{\gamma} \alpha} M_{\dot{\beta}}^{\dot{\gamma}}+4 i H_{\dot{\beta} \alpha} Y, \\
{\left[\nabla_{\alpha}, \nabla_{b}\right]=} & -2 \nabla_{\beta} H_{\dot{\beta} \gamma} M_{\alpha}^{\gamma} \\
& +\left[-2 i C_{\alpha \beta} \bar{W}_{\dot{\beta} \dot{\gamma}}^{\dot{\delta}}+C_{\dot{\beta} \dot{\gamma}}\left(\nabla_{(\alpha} H^{\dot{\delta}}{ }_{\beta)}-\frac{1}{3} C_{\alpha \beta} \nabla^{\epsilon} H^{\dot{\delta}}{ }_{\epsilon}\right)\right] M_{\dot{\delta}} \dot{\gamma} \\
& +2 \nabla_{\beta} H_{\dot{\beta} \alpha} Y \\
{\left[\nabla_{a}, \nabla_{b}\right]=} & -2 H_{\dot{\alpha} \beta} \nabla_{\alpha \dot{\beta}} \\
& +\left[\frac{i}{2} C_{\alpha \beta} \nabla_{(\dot{\alpha}} H_{\dot{\beta})}{ }^{\gamma}+C_{\dot{\alpha} \dot{\beta}}\left(-\frac{i}{6} C^{\gamma}{ }_{(\alpha \mid} \nabla^{\dot{\epsilon}} H_{\dot{\epsilon} \mid \beta)}+W_{\alpha \beta}{ }^{\gamma}\right)\right] \nabla_{\gamma} \\
& +\left[C _ { \dot { \alpha } \dot { \beta } } \left(\frac{1}{24} \nabla_{(\alpha} W_{\beta \gamma}{ }^{\delta)}+\frac{1}{4}\left(C^{\delta}{ }_{\alpha} \nabla_{(\beta \mid \dot{\epsilon}} H^{\dot{\epsilon}} \mid \gamma\right)\right.\right. \\
& \left.\left.+\frac{i}{6} C_{\alpha \gamma} C^{\delta}{ }_{\beta} \nabla^{\dot{\epsilon}} \nabla^{\epsilon} H_{\dot{\epsilon} \epsilon}\right)+\frac{i}{2} C_{\alpha \beta} \nabla_{\gamma} \nabla_{(\dot{\alpha}} H_{\dot{\beta})}{ }^{\delta}\right] M_{\delta}{ }^{\gamma} \\
& \left.-\frac{i}{2} C_{\alpha \beta} \nabla^{\delta} \nabla_{(\dot{\alpha}} H_{\dot{\beta}) \delta} Y+\text { c.c. }\right\}
\end{aligned}
$$

where $Y$ and $M_{A}{ }^{B}$ are the generators of $U(1)$ and Lorentz transformations, and $W_{\alpha \beta \gamma}$ is completely symmetric chiral superfield,

$$
\nabla_{\dot{\delta}} W_{\alpha \beta \gamma}=0,
$$


and $H_{\dot{\alpha} \beta}$ is defined as follows in terms of the field strength $H_{A B C}$ of $B_{A B}\left(C_{\alpha \beta}\right.$ is proportional to the Pauli matrix $\sigma_{2}$ and used to raise and lower indices),

$$
H_{a b c}=C_{\gamma \alpha} C_{\dot{\gamma} \dot{\beta}} H_{\dot{\alpha} \beta}-C_{\gamma \beta} C_{\dot{\gamma} \dot{\alpha}} H_{\dot{\beta} \alpha} .
$$

$W_{\alpha \beta \gamma}$ and $H_{\dot{\alpha} \beta}$ satisfy the following differential relations

$$
\nabla_{a} H^{a}=0, \nabla^{\gamma} W_{\gamma \alpha \beta}=\frac{i}{6} \nabla_{(\alpha \mid} \nabla^{\dot{\gamma}} H_{\dot{\gamma} \mid \beta)}+\frac{i}{2} \nabla^{\dot{\gamma}} \nabla_{(\alpha \mid} H_{\dot{\gamma} \mid \beta)}, \quad \nabla^{\beta} \nabla_{\beta} H_{a}=0 .
$$

Furthermore, all components of the field strength $H_{A B C}$ vanish. except $H_{\alpha \dot{\beta} c}$ and $H_{a b c}$. A similar supergravity algebra has been obtained in 11 .

At one-loop we found a set of field equations and the action that yields them. All equations do in fact follow by requiring that the OPE of $T$ with itself is correct through one loop. This implies that we can also obtain the field equations by a conventional beta-function calculation. It is not clear whether this is also true at higher loop, but if true would dramatically simplify the analysis of higher order corrections to the field equations.

\section{Conventional $\beta$-function calculation}

For simplicity, we take only the first term of (11) and couple it to an arbitrary world-sheet metric $g_{i j}$,

$$
S=\frac{1}{8 \alpha^{\prime}} \int d^{2} z \sqrt{g} g^{i j} \partial_{i} z^{M} E_{M}{ }^{a} \partial_{j} z^{N} E_{N}{ }^{a}+\frac{1}{8} \int d^{2} z \sqrt{g} R(\phi+\bar{\phi})
$$

Next, we take the background metric purely conformal, $g_{i j}=e^{\sigma} \delta_{i j}$, and write the action in $2-2 \epsilon$ dimensions,

$$
\begin{aligned}
S=\frac{1}{8 \alpha^{\prime}} \int d^{2-2 \epsilon} z e^{-\epsilon \sigma} \delta^{i j}\left(\partial_{i} z^{M} E_{M}{ }^{a} \partial_{j} z^{N} E_{N}{ }^{a}\right. \\
\left.+\alpha^{\prime}(\phi+\bar{\phi})\left((1-2 \epsilon) \partial_{i} \partial_{j} \sigma-\frac{\epsilon}{2}(1-2 \epsilon) \partial_{i} \sigma \partial_{j} \sigma\right)\right) .
\end{aligned}
$$

Conformal invariance means that the action should be independent of $\sigma$. Classically, when the dilaton terms do not contribute, this is obvious by taking $\epsilon \rightarrow 0$. At one-loop, there are counterterms that have to be added to the action in order to make the path integral finite. Such counterterms are needed to cancel UV divergences. The only UV divergences at one-loop come from terms in the background field expansion of the action of the form $\int d^{2} z y^{a} y^{b} C_{b a}$, where $y^{a}$ represents a quantum fluctuation of $z^{M}$ and $C_{b a}$ depends only on the background fields. Contracting the fields $y^{a}$ and $y^{b}$ yields a momentum space 
integral of the form $\int d^{2} k|k|^{-2}$. In principle, in dimensional regularization, this integral vanishes, as the IR and UV divergences cancel each other. However, string theory should properly be defined on a compact world-sheet in which case there should be no IR divergence. Therefore, the IR divergence should be subtracted out, yielding $2 \int d^{2} k\left(|k|^{-2}+\frac{1}{\epsilon} \delta^{(2)}(k)\right)=\frac{1}{\epsilon}$. This shows that we have to add to $\int d^{2} z y^{a} y^{b} C_{b a}$ the counterterm $-\int d^{2} z \frac{\alpha^{\prime}}{\epsilon} C_{a a}$. Inserting this in (11) we find a contribution

$$
S=\frac{1}{\alpha^{\prime}} \int d^{2-2 \epsilon} z e^{-\epsilon \sigma}\left(y^{a} y^{b} C_{a b}-\frac{\alpha^{\prime}}{\epsilon} C_{a a}+\frac{\alpha^{\prime}}{8}(\phi+\bar{\phi}) \partial_{i} \partial_{j} \sigma+\mathcal{O}(\epsilon)\right) .
$$

If we take the derivative of this action with respect to sigma and then take $\epsilon \rightarrow 0$, we obtain a nontrivial identity

$$
C_{a a}+\frac{1}{2} \partial \bar{\partial}(\phi+\bar{\phi})=0 .
$$

If we extract $C_{a a}$ from the background field expansion of the sigma model, and manipulate (13) using the equations of motion of the sigma model and the supergravity algebra (3)-(河), we find three equations. These are not all independent, but are all consequences of just one which reads

$$
2 \nabla_{\alpha} H_{\dot{\alpha} \beta}=\nabla_{\beta} \nabla_{a}(\phi+\bar{\phi}) .
$$

This equation of motion can be derived from the action

$$
S=\int d^{4} x d^{4} \theta E^{-1} e^{\phi+\bar{\phi}}
$$

which is the low-energy effective action of supergravity coupled to a tensor multiplet $G$, in the gauge where $G=1$. One can verify that the kinetic term of $\phi$ and $\bar{\phi}$ is negative in (15), showing that the dilaton is part of the compensator. Thus, the compensator consists of a chiral and anti-chiral multiplet and we are dealing with old-minimal supergravity.

\section{Conclusions}

We have seen how the tree-level and one-loop analysis determine the off-shell formulation of supergravity corresponding to the heterotic string. The antisymmetric tensor is part of a tensor multiplet with reduced field strength $G$, and the sigma model automatically selects the 'string gauge' $G=1$. One could reintroduce the tensor multiplet, leading to the conformally invariant action

$$
S=\int d^{4} x d^{4} \theta E^{-1} G^{-\frac{1}{2}} e^{\phi+\bar{\phi}} .
$$


Thus, the most natural framework for string phenomology is one involving matter in a tensor multiplet (properties of such actions have been studied in the literature 13 ), with a chiral and anti-chiral compensator. However, one should be careful not to identify the lowest component of this tensor multiplet with the dilaton.

Besides this, it would be interesting to include the Yang-Mills and compactification dependent fields in the discussion, and to analyze the type II string. In addition, we would like to understand whether the standard betafunction calculation is always sufficient, and whether it is possible to derive the low-energy effective action directly from the sigma model as can be done for the bosonic string 4 .

We also hope to apply these supersymmetric sigma models to other cases, like the $(1,2)$ heterotic strings 1 , and have indications that it may be possible to find a sigma model describing self-dual supergravity $\mathrm{g}$.

\section{References}

1. M. Green, Nucl. Phys. B 293, 593 (1987); D. Kutasov and E. Martinec, Nucl. Phys. B 477, 652 (1996); D. Kutasov, E. Martinec and M. O'Loughlin, Nucl. Phys. B 477, 675 (1996); E. Martinec, Geometrical Structure of $M$ Theory, EFI-96-29, hep-th/9608017.

2. M. B. Green and J. H. Schwarz, Nucl. Phys. B 243, 285 (1984).

3. N. Berkovits, Nucl. Phys. B 395, 77 (1993); N. Berkovits, Nucl. Phys. B 420, 332 (1994); N. Berkovits, Nucl. Phys. B 431, 258 (1994).

4. N. Berkovits and W. Siegel, Nucl. Phys. B 462, 213 (1996).

5. W. Siegel, Phys. Rev. D 53, 3336 (1996).

6. J. de Boer and K. Skenderis, to appear in Nucl. Phys. B.

7. S. Cecotti, S. Ferrara and M. Villasante, Int. J. Mod. Phys. A 2, 1839 (1987); J.-P. Derendinger, F. Quevedo and M. Quirós, Nucl. Phys. B 428, 282 (1994).

8. J. de Boer and K. Skenderis, in preparation.

9. E.S. Fradkin and A.A. Tseytlin, Phys. Lett. B 158, 316 (1985); Nucl. Phys. B 261, 1 (1985).

10. T. Banks, D. Nemeschansky and A. Sen, Nucl. Phys. B 277, 67 (1986).

11. S. Gates, P. Majumdar, R. Oerter and E. van de Ven, Phys. Lett. B 214, 26 (1988).

12. B. de Wit, M.T. Grisaru and P. van Nieuwenhuizen, Nucl. Phys. B 408, 299 (1993).

13. P. Binétruy, M.K. Gaillard and Y.-Y. Wu, Dilaton Stabilization in the Context of Dynamical Supersymmetry Breaking through Gaugino Condensation, hepth/9605170.

14. O.D. Andreev, R.R. Metsaev and A.A. Tseytlin, Sov. J. Nucl. Phys. 51, 359 (1990). 